\title{
Piezoelectric shunt damping by synchronized switching on negative capacitance and adaptive voltage sources
}

\author{
Ehtesham Mustafa Qureshi*, Xing Shen** and JinJin Chen*** \\ College of Aerospace Engineering, State Key Laboratory of Mechanics and Control of Mechanical Structures, Nanjing University \\ of Aeronautics and Astronautics, No. 29 Yudao Street, Nanjing 210016, China.
}

\begin{abstract}
Synchronized switch damping (SSD) techniques have recently been developed for structural vibration control using piezoelectric materials. In these techniques, piezoelectric materials are bonded on the vibrating structure and shunted by a network of electrical elements. These piezoelectric materials are switched according to the amplitude of the excitation force to damp vibration. This paper presents a new SSD technique called 'synchronized switch damping on negative capacitance and adaptive voltage sources' (SSDNCAV). The technique combines the phenomenon of capacitance transient charging and electrical resonance to effectively dampen the structural vibration. Also, the problem of stability observed in the previous SSD techniques is effectively addressed by adapting the voltage on the piezoelectric patch according to the vibration amplitude of the structure. Analytical expressions of vibration attenuation at the resonance frequency are derived, and the effectiveness of this new technique is demonstrated, for the control of a resonant cantilever beam with bonded piezoelectric patches, by comparing with SSDI, SSDVenh, and SSDNC techniques. Theoretical predictions and experimental results show the remarkable vibration damping capability of SSDNCAV technique, which was better than the previous SSD techniques. The broadband vibration control capabilities of SSDNCAV technique are also demonstrated, which exceed those of previous SSD techniques.
\end{abstract}

Key words: damping, piezoelectric, shunt, switch

\section{Introduction}

Structural vibration control using piezoelectric materials is an established field of study. These materials, due to their small volume, lightweight, mechanical simplicity, and ease of integration with mechanical structures, have emerged as materials of choice among various researchers and engineers. Their precise positioning and dynamics also makes them suitable for low frequency vibration damping applications. Piezoelectric shunt damping is an alternative to active vibration control [1]. These techniques have effectively been used over the last two decades for structural vibration control. They involve integrating piezoelectric patches on the vibrating structure and connecting it to a network of electrical elements. Vibration energy is then converted into electrical energy by the direct piezoelectric effect and dissipated through electric circuits [2-4].

Vibration control using piezoelectric shunt can be categorized into passive, semi-passive and semi-active. Although some researchers have used both terms ("semipassive" and "semi-active") to indicate different shunt damping circuits with an external power supply, e.g., Qiu et al. [5], according to Wang et al. [6], an electric shunt circuit is taken as semi-passive, if and only if it does not provide power to the system; otherwise, it is considered semi-active. Passive shunt damping, which is the simplest of the control techniques, consists of a piezoelectric patch shunted by a resistive-inductive RL electric network [3-4, 7-10]. However, variation in system parameters, which may be due to environmental effects or change of external loads,
This is an Open Access article distributed under the terms of the Creative Commons Attribution Non-Commercial License (http://creativecommons.org/licenses/by$\mathrm{nc} / 3.0 /$ which permits unrestricted non-commercial use, distribution, and reproduction in any medium, provided the original work is properly cited. (c) * Ph. D Candidate
** Professor, Corresponding author: shenx@nuaa.edu.cn
** Graduate Student 
causes degradation in their control performance. Also, the requirement for high shunt impedances for low frequency vibration control and their narrow band behavior makes these systems unattractive for onboard applications, such as aerospace. To overcome these disadvantages, semi-passive and semi-active techniques have been developed, which have proved to be effective vibration control systems [1126].

A class of semi-passive and semi-active vibration control techniques called 'synchronized switch damping' (SSD), has become popular for its effectiveness in low frequency vibration control, insensitivity towards resonance frequency variation, and enhanced damping performance over a large frequency bandwidth. These are basically non-linear switching techniques in which the piezoelectric voltage is inverted repeatedly synchronously with the structure motion [11-13]. Vibration attenuation is achieved by adaptively tuning the shunt parameters to match the resonance frequencies of the vibrating structure. There are several variants of SSD systems. The simplest is synchronized switch damping on a resistance (SSDS) [11]. In this technique, the voltage on the piezoelectric patch attached to the vibrating structure, which is the image of the structure's displacement, is switched to zero when the displacement reaches a predefined threshold. The switching action is performed using a pair of solid state switches driven by a controlled width pulse, generated by a simple microcontroller. An improvement in this technique was made by Richard et al. [13] by including an inductance in the shunt circuit, which became known as 'synchronized switch damping on inductance' (SSDI). An inductor in the shunt circuit and the inherent capacitance of piezoelectric patch give rise to electrical resonance, which, if properly tuned, allows the inversion and magnification of piezoelectric voltage. Also, a phase shift is induced between the piezoelectric strain and the resulting voltage, thus creating energy dissipation and consequently increasing the damping performance.

Thus, damping performance has a direct relationship with the magnitude of the voltage on the piezoelectric patch. Also, this voltage magnitude is dependent on the quality factor of the shunt circuit $[14,18]$. However, it is practically difficult to increase the quality factor beyond a certain limit, which is a disadvantage of SSDI techniques $[19,27]$. The principle that the higher the magnitude of voltage on the piezoelectric patch, the higher is the damping performance resulted in another SSD technique called 'synchronized switch damping on voltage sources' (SSDV) [19]. The addition of constant voltage sources in the shunt circuit increases the magnitude of voltage on the piezoelectric patch, thus enhancing the damping performance. However, a stability problem is observed when the vibration level is very low because these constant voltage sources may excite the structure instead of attenuating it. To compensate for the stability problem, an enhancement was proposed by Badel et al. [20], which consisted of adapting the voltage sources to the amplitude of vibration. A more recent work on this technique can be found in Ji et al. [22, 28].

Ji et al. [27] proposed a new technique called 'synchronized switch damping on negative capacitance' (SSDNC) by replacing the inductance in the SSDI technique with a negative capacitance. The use of negative capacitance makes the whole circuit capacitive, without resonance. However, voltage inversion on the piezoelectric patch is still possible. The magnitude of the voltage on the piezoelectric patch depends on the ratio of inherent capacitance of the piezoelectric patch and the negative capacitance. Thus, 'good' damping performance is achieved by appropriately choosing the value of negative capacitance, which magnifies the voltage on the piezoelectric patch. Mokrani et al. [29] attempted to combine both the adaptive nature of SSDI and enhanced damping performance of SSDNC into a technique called 'synchronized switch damping on negative capacitance and inductance' (SSDNCI). The problem of synthetic negative capacitor saturation, due to the high current resulting from closing the switch, was effectively solved by limiting the amount of current that is supplied by the negative capacitance, through the use of an inductor. This improved the damping performance relative to SSDNC. A more recent version of this technique was proposed by Han et al. [30].

This paper proposes a new technique called 'synchronized switch damping on negative capacitance and adaptive voltage sources' (SSDNCAV). An improvement in damping performance is achieved by combining the effect of negative capacitance with electrical resonance in the switching shunt circuit, along with the adaptive adjustment of voltage sources according to the vibration amplitude. First, a theoretical analysis of the SSD techniques is presented, showing the influence of the electrical quality factor of the shunt circuit on the damping performance. Then, experiments are performed on a cantilever beam to validate the theoretical results and confirm the effectiveness of the proposed SSDNCAV technique compared with other SSD techniques for single-mode vibration control. Finally, the vibration-damping capability of the SSDNCAV technique is demonstrated under broadband excitation by exciting the cantilever beam by a short sine sweep signal at a $500 \mathrm{~Hz}$ frequency band. 


\section{Modeling}

The electromechanical behavior of a vibrating structure containing a piezoelectric patch can be modeled as a springmass-damper system as shown in Fig. 1. In its simplest form, the structure can be modeled as a second-order model if it is driven around one of its resonance frequencies under harmonic excitation [20]. Assuming linear elastic properties for the structure and the piezoelectric patch, differential Eq. (1) can be established,

$$
M \ddot{u}+C \dot{u}+K_{E} u=\sum F_{i}
$$

where $M$ represents the equivalent rigid mass and $C$ is the mechanical loss coefficient, $K_{E}$ is the equivalent stiffness of the mechanical structure and piezoelectric patch in a shortcircuited condition, $u$ is the rigid mass displacement, and $\Sigma F_{i}$ represents the sum of all other forces applied to equivalent rigid mass including forces applied by the piezoelectric patch.

Eqs. (2) and (3) describe the electromechanical coupling of piezoelectric patch bonded on the vibrating structure,

$$
\begin{aligned}
& F_{p}=-\alpha V_{p} \\
& I_{p}=\alpha \dot{u}-C_{p} \dot{V}_{p}
\end{aligned}
$$

where $F_{p}$ is the electrically dependent part of the force applied by the piezoelectric patch on the structure, $\alpha$ is the force factor, $C_{p}$ is the blocked capacitance of piezoelectric patch, and $I_{p}$ is the outgoing current from piezoelectric patch. $M, K_{E}, \alpha$ and $C_{p}$ can be determined from the structure and piezoelectric patch characteristics and geometry. As $\Sigma F_{i}$ is the combination of $F_{p}$ and the external applied force on the structure (i.e., the excitation force $F$ ). Thus, Eq. (1) can be written as,

$$
M \ddot{u}+C \dot{u}+K_{E} u=F-\alpha V_{p}
$$

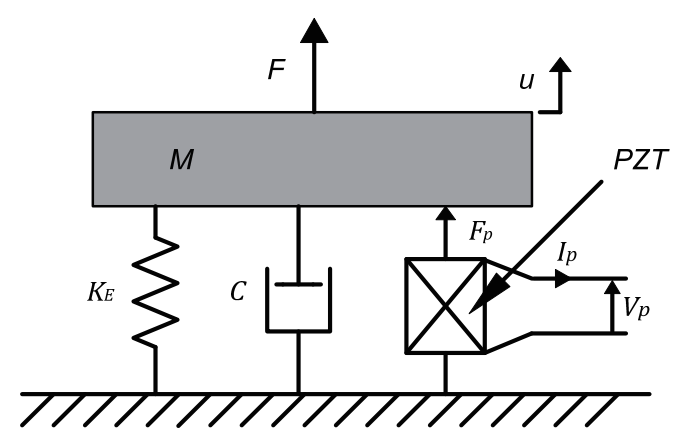

Fig. 1. Schematic representation of the electromechanical model for piezoelectric shunt damping. PZT: piezoelectric patch. which represents the differential equation of motion of the electromechanical vibration system.

Multiplying both sides of Eq. (4) by velocity and integrating over the time variable gives the following energy Eq. (5),

$$
\left\{\int F \dot{u} d t=\frac{1}{2} M \dot{u}^{2}+\frac{1}{2} K_{E} u^{2}+\int C \dot{u}^{2} d t+\int \alpha V_{p} \dot{u} d t\right\}
$$

which represents the provided energy in terms of kinetic energy, potential energy, mechanical losses, and transferred energy. Here, the transferred energy represents conversion of a part of the mechanical energy to electrical energy. By maximizing this energy, the mechanical energy corresponding to kinetic and potential energy in the structure can be minimized.

If nothing is connected to the piezoelectric patch (i.e., the open circuit condition), then the transferred energy is clearly equal to zero because the piezoelectric voltage $V_{p}$ and velocity $\dot{u}$ are in phase. Thus, the output current of the piezoelectric patch also becomes zero. This condition, when applied to Eq. (3), leads us to write Eq. (6) in the frequency domain as,

$$
\tilde{V}_{p}=\frac{\alpha}{C_{p}} \tilde{u}
$$

Writing Eq. (4) in the frequency domain and inserting Eq. (6) into it, leads us to the transfer function (7) linking the displacement to the excitation force,

$$
\frac{\widetilde{u}}{\widetilde{F}}=\frac{1}{\left(K_{E}+\frac{\alpha^{2}}{C_{p}}-M \omega^{2}+j C \omega\right)}
$$

Under the assumption that at resonance frequency, the force $F$ and the velocity $\dot{u}$ are in phase [20], the resonance angular frequency is given by Eq. (8):

$$
\omega_{o}=\sqrt{\frac{K_{E}+\frac{\alpha^{2}}{C_{p}}}{M}}
$$

In the case of the open circuit condition, the amplitude of vibration is only limited by the inherent structural damping of the structure, as shown in Eq. (9), where $F_{M}$ is the amplitude of the excitation force and $\omega_{\mathrm{o}}$ is the angular resonance frequency. Under the closed circuit condition, the expression of vibration attenuation, represented by the performance index $A_{p}$ in $\mathrm{dB}$, is given by Eq. (10), where $U_{M_{0}}$ and $U_{M}$ are the open and closed circuit vibration amplitudes, respectively.

$$
\begin{aligned}
& U_{M_{o}}=\frac{F_{M}}{C \omega_{o}} \\
& A_{p}=20 \log \left(\frac{U_{M}}{U_{M_{o}}}\right)
\end{aligned}
$$




\subsection{SSD on Inductance (SSDI)}

The SSDI technique is represented schematically in Fig. 2. It consists of an electronic switch, a resistor $R$ and an inductor $L$, all connected in series. Closing the switch at the point of maximum displacement $u$, which corresponds to the maximum voltage on the piezoelectric patch, leads to the pseudo-periodic voltage oscillation, until the switch is open [18]. Thus, the piezoelectric patch voltage $V_{p}$ is amplified, time-shifted, and distorted as shown in Fig. 3. The switch is kept closed until the voltage $V_{p}$ on the piezoelectric patch is reversed. This corresponds to the time $t_{i}$ equal to half the pseudo-period of the electric $L R C_{p}$ circuit, given in Eq. (11):

$$
t_{i}=\pi \sqrt{L C_{p}}
$$

The voltage and the displacement vary proportionally when the switch is open and no load is connected. The inversion of voltage is not perfect, because part of the energy stored on the piezoelectric patch capacitance is lost in the switching network consisting of an electronic switch, resistor, and inductor. These losses are modeled by an electrical quality factor $Q_{i}[20]$. The relationship between $Q_{i}$ and the piezoelectric patch voltage, before and after the inversion process is given in Eq. (12),

$$
V_{m}=V_{M} e^{\frac{-\pi}{2 Q_{i}}}=\gamma V_{M}
$$

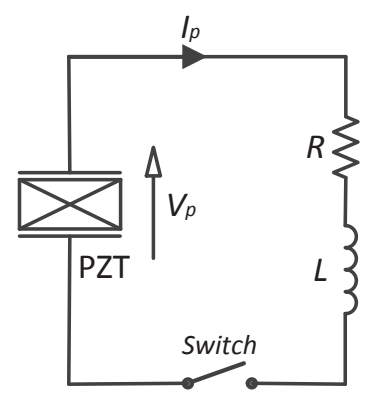

Fig. 2. Schematic diagram of SSDI. PZT: piezoelectric patch.

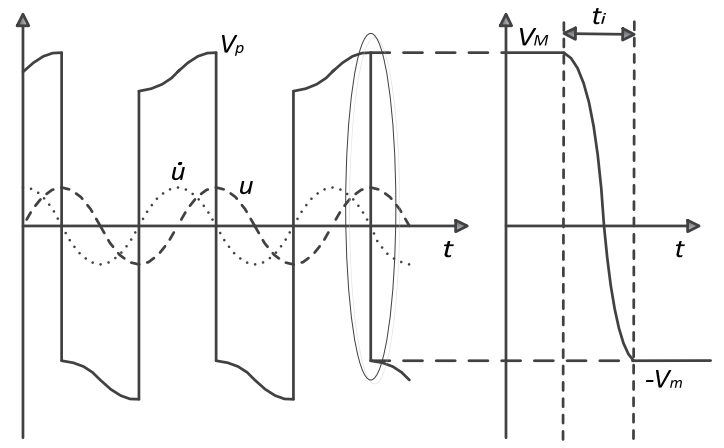

Fig. 3. Piezoelectric patch voltage and the structural displacement. where $V_{M}$ and $V_{m}$ are the piezoelectric patch voltages before and after the inversion process respectively and $\gamma$ is the voltage inversion coefficient. The electrical quality factor $Q_{i}$ of the $L R C_{p}$ circuit is given in Eq. (13):

$$
Q_{i}=\frac{1}{R} \sqrt{\frac{L}{C_{p}}}
$$

The transferred energy can be expressed as the following integral function of voltage $V_{p}$ and displacement $u$ :

$$
\int \alpha V_{p} \dot{u} d t=\int \alpha V_{p} d u
$$

The transferred energy during the whole process can be represented by an energy cycle as shown in Fig. 4, under the condition that the structure motion is periodic. This energy cycle clearly represents two open circuit phases and two closed circuit or voltage inversion phases. In the open circuit, the piezoelectric patch voltage is proportional to displacement with a factor $\alpha / C_{p}$. However, in the voltage inversion phases, no displacement is observed as the inversion time is much shorter than the mechanical motion period. The surface circumscribed by this cycle represents the image of the transferred energy with a factor $1 / \alpha$. The voltage and the displacement vary proportionally between two voltage inversion, leading to Eq. (15), where $u_{M}$ is the displacement amplitude. The transferred energy $E_{t}$ during the period is given by Eq. (16):

$$
\begin{aligned}
& V_{M}=V_{m}+\frac{2 \alpha}{C_{p}} u_{M} \\
& \left\{E_{t}=\int_{\text {period }} \alpha V_{p} d u=2 \alpha u_{M}\left(V_{m}+V_{M}\right)\right\}
\end{aligned}
$$

From Eqs. (12), (15), and (16), $E_{t}$ can be expressed as a unique function of the displacement amplitude, as shown in Eq. (17), while the theoretical value of the SSDI damping is given by Eq. (18) [20]:

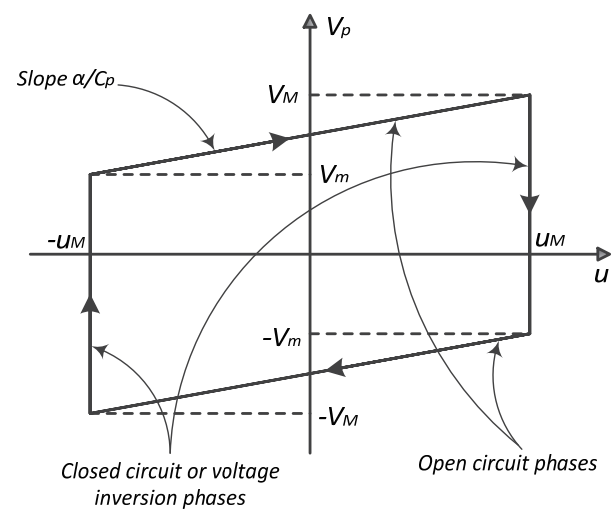

Fig. 4. Energy cycle showing open and closed circuit phases. 


$$
\begin{aligned}
& E_{t}=\frac{4 \alpha^{2}}{C_{p}} \frac{1+\gamma}{1-\gamma} u_{M}^{2} \\
& \left\{A_{S S D I}=20 \log \left(\frac{C \omega_{o}}{C \omega_{o}+\left(4 \alpha^{2} / \pi C_{p}\right)(1+\gamma) /(1-\gamma)}\right)\right\}
\end{aligned}
$$

From Eqs. (13), (17), and (18), it is clear that the electrical quality factor $Q_{i}$ influences the electromechancial transferred energy and the damping effect. As discussed by Lefeuvre et al. [19], $Q_{i}$ decreases as the resistor $R$ increases. As $R$ in the circuit induces loss of energy during the inversion process, it limits the amplitude of piezoelectric patch voltage.

\subsection{SSD on Voltage Sources (SSDV)}

A schematic representation of the SSDV technique is shown in Fig. 5. The additional voltage sources $V_{C C}$ increases the inverted voltage on the piezoelectric patch, compared with the SSDI technique, thus increasing the dissipated energy and consequently the damping effect. A typical time history plot of piezoelectric voltage and structural displacement is shown in Fig. 6.

The voltage variations between the two voltage inversions still lead to Eq. (15) but considering the effect of the voltage sources $V_{C C}$, the voltage inversion is given by Eq. (19). The dissipated energy $E_{t}$ expressed as a function of $u_{M}$ and $V_{C C}$ is

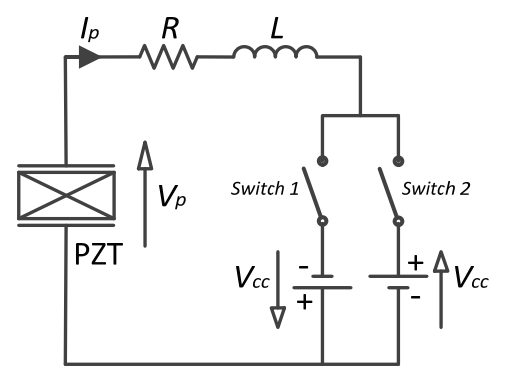

Fig. 5. Schematic diagram of SSDV. PZT: piezoelectric patch.

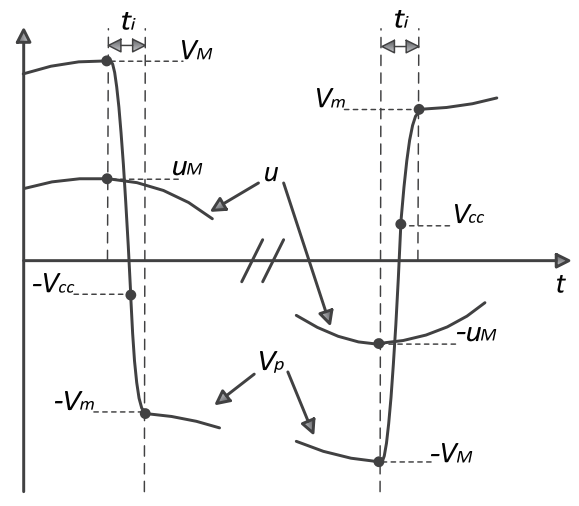

Fig. 6. Voltage on the piezoelectric patch and the structural displacement. as shown in Eq. (20) [20]:

$$
\begin{aligned}
& V_{m}-V_{c c}=\gamma\left(V_{M}+V_{c c}\right) \\
& E_{t}=\left(\frac{4 \alpha^{2}}{c_{p}} u_{M}^{2}+4 \alpha u_{M} V_{c c}\right) \frac{1+\gamma}{1-\gamma}
\end{aligned}
$$

The theoretical value of the SSDV damping is given by Eq. (21),

$$
\left\{A_{S S D V}=20 \log \left(\frac{C \omega_{o}}{C \omega_{o}+\left(4 \alpha^{2} / \pi c_{p}\right)(1+\gamma) /(1-\gamma)}\right) \times\left(1-\frac{4}{\pi} \frac{1+\gamma}{1-\gamma} \frac{\alpha V_{c c}}{F_{M}}\right)\right\}
$$

where $F_{M}$ is the amplitude of the excitation force $F$. Thus, the SSDV technique gives better damping performance than SSDI, but the problem of stability is observed due to the use of constant voltage sources. This leads the piezoelectric voltage to have a driving effect, instead of a braking effect. Eq. (21) shows that a value of voltage $V_{C C}$, which totally cancels the vibration, under a given excitation force, can be found. This value is given as:

$$
V_{c c \max }=\frac{\pi}{4 \alpha} \frac{1-\gamma}{1+\gamma} F_{M}
$$

This is the maximum voltage that can be applied under the given excitation force. Applying a voltage higher than $V_{\text {CCmax }}$ causes system instability.

\subsection{Enhanced SSDV (SSDVenh)}

As shown in Eq. (22), the voltage source is directly proportional to the amplitude of the excitation force. If this voltage source can be adapted to the vibration amplitude, the problem of stability can be solved [20]. The schematic diagram of enhanced SSDV is shown in Fig. 7. In the enhanced SSDV, the voltage source is proportional to vibration amplitude; that is:

$$
V_{c c}=-\beta \frac{\alpha}{C_{p}} u
$$

$$
\left\{V_{c c}=-\beta \frac{\alpha}{C_{p}} u_{M} \text { on strain maximum }\right\},
$$

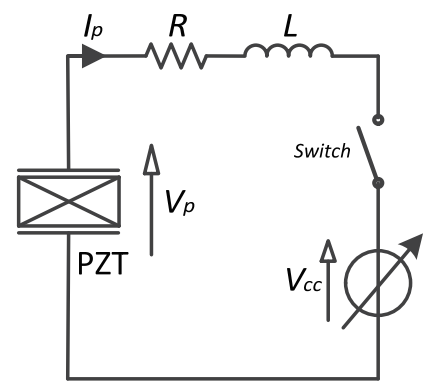

Fig. 7. Schematic diagram of SSDVenh. PZT: piezoelectric patch. 


$$
\left\{V_{c c}=\beta \frac{\alpha}{C_{p}} u_{M} \quad \text { on strain minimum }\right\}
$$

where $\beta$ is the proportional coefficient set by the user. The voltage variations between the two voltage inversions still lead to Eq. (15), but considering the effect of the voltage sources $V_{C C}$, the voltage inversion is given by Eq. (26). The dissipated energy $E_{t}$ during a period can be expressed as in Eq. (27) [20]:

$$
\begin{aligned}
& V_{m}-\beta \frac{\alpha}{c_{p}} u_{M}=\gamma\left(V_{M}+\beta \frac{\alpha}{c_{p}} u_{M}\right) \\
& E_{t}=\frac{4 \alpha^{2}}{c_{p}}(1+\beta) \frac{1+\gamma}{1-\gamma} u_{M}^{2}
\end{aligned}
$$

As compared to the original SSDV technique, the enhanced SSDV technique increases the dissipated energy, thus increasing the vibration damping. The theoretical value of the enhanced SSDV damping is given by Eq. (28):

$$
\left\{A_{S S D V_{\text {enh }}}=20 \log \left(\frac{C \omega_{o}}{C \omega_{o}+\left(4 \alpha^{2} / \pi C_{p}\right)(1+\beta)(1+\gamma) /(1-\gamma)}\right)\right\} .
$$

This equation shows that for a given value of $\beta$, the damping is insensitive to the amplitude of the applied force. This is the critical point of the enhanced SSDV technique. Also, it is to be noted that higher values of parameter $\beta$ render the previous theoretical developments invalid, because the displacement of higher-order modes cannot be neglected as compared to the fundamental one.

\subsection{SSD on Negative Capacitance (SSDNC)}

The schematic representation of SSDNC is shown in Fig. 8 in which a negative capacitance $-C_{n}$ replaces an inductor in SSDI. A voltage inversion on the piezoelectric patch is still possible even in the absence of resonance. This inverted voltage $V_{m}$ is given by the following Eq. (29) [27],

$$
V_{m}=-\frac{C_{p}}{C_{n}-C_{p}} \frac{\alpha}{C_{p}} u_{M}
$$

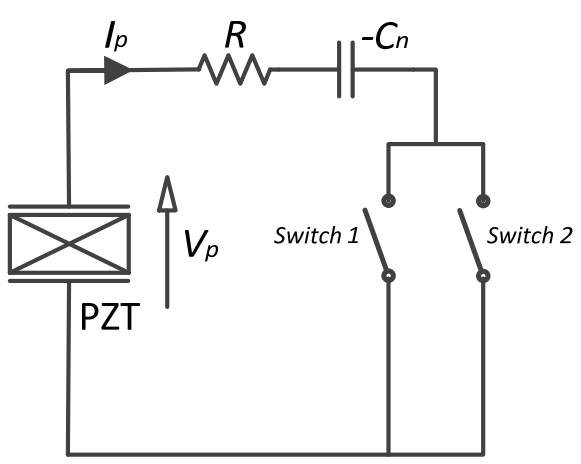

Fig. 8. Schematic diagram of SSDNC. PZT: piezoelectric patch. which shows that the voltage on the piezoelectric patch is inverted and magnified by a factor of $C_{p} /\left(C_{n}-C_{p}\right)$. This equation also shows that the magnitude of voltage on the piezoelectric patch is independent of the electrical quality factor of the circuit as compared to the SSDI and SSDV techniques. The waveform of voltage on the piezoelectric patch is shown in Fig. 9.

Between two voltage inversions, the voltage and displacement vary proportionally according to Eq. (30), while the transferred energy is given by Eq. (31):

$$
\begin{aligned}
& V_{M}+V_{m}=\frac{C_{n}}{C_{n}-C_{p}} \frac{2 \alpha}{C_{p}} u_{M} \\
& E_{t}=\frac{C_{n}}{C_{n}-C_{p}} \frac{4 \alpha^{2}}{C_{p}} u_{M}^{2}
\end{aligned}
$$

In the SSDNC technique, the voltage on the piezoelectric patch depends on the capacitance ratio $C_{n} / C_{p}$, while in SSDI technique, this voltage is dependent on the electrical quality factor of the circuit. Because it is difficult to fabricate an inductor with a high quality factor, the voltage on the piezoelectric patch in SSDI is limited. However, in SSDNC this voltage can be increased readily by choosing an appropriate value of negative capacitance [30]. The theoretical value of the SSDNC damping is given by Eq. (32):

$$
\left\{A_{S S D N C}=20 \log \left(\frac{C \omega_{o}}{C \omega_{o}+\left(4 \alpha^{2} / \pi C_{p}\right)\left(C_{n} /\left(C_{n}-C_{p}\right)\right)}\right)\right\}
$$

\subsection{SSD on Negative Capacitance and Adaptive Voltage Source (SSDNCAV)}

The new technique proposed in this paper, called the 'synchronized switch damping on negative capacitance and adaptive voltage source' (SSDNCAV), combines the effect of both SSDVenh and SSDNC to further increase the voltage of piezoelectric patch for enhanced damping performance.

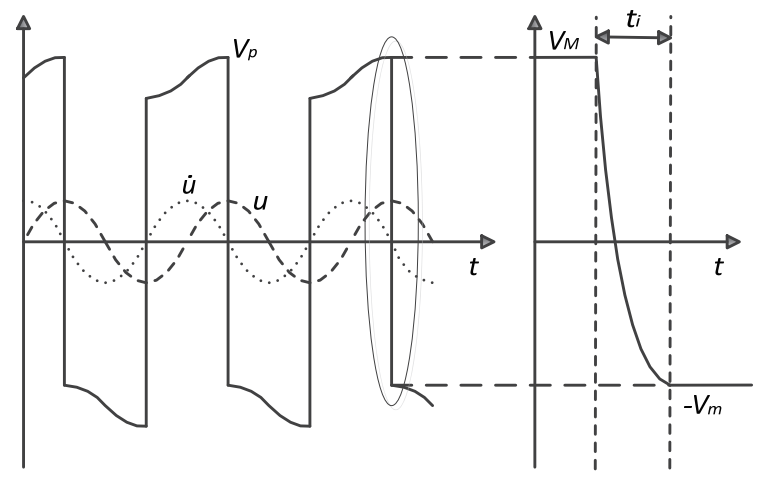

Fig. 9. Waveform of voltage on the piezoelectric patch in SSDNC. 
Voltage inversion on the piezoelectric patch takes place via the capacitance transient charging (in SSDNC) and circuit oscillation due to an $L C_{p}$ resonance (in SSDVenh). Also, the voltage on the piezoelectric patch is adapted according to the vibration amplitude of the structure, thus reducing the problem of instability as observed in previous SSD techniques. The vibration amplitude is monitored using an additional piezoelectric patch, bonded in the same area as the piezoelectric patch used for controlling the vibration. Considering a model similar to Fig. 1, the voltage on the additional piezoelectric patch is given by Eq. (33) and $V_{C C}$ in Eq. (34), while $\beta$ is the proportional coefficient, as described earlier, which needs to be set by user. The schematic diagram of SSDNCAV is shown in Fig. 10.

Similar to the previous SSD techniques, the control strategy consists of closing the switch when the extremum of displacement occurs, corresponding to the extremum of voltage on the piezoelectric patch $V_{p}$. The switch is open after the voltage inversion takes place. Considering sinusoidal steady-state excitation, the voltage $V_{C C}$ during the voltage inversion is given by Eqs. (35) and (36).

$$
\begin{aligned}
& V_{S}=\frac{\alpha}{C_{p}} u \\
& V_{c c}=-\beta \frac{C_{p}}{C_{n}-C_{p}} \frac{\alpha}{C_{p}} u \\
& \left\{V_{c c}=-\beta \frac{C_{p}}{C_{n}-C_{p}} \frac{\alpha}{C_{p}} u_{M} \text { on strain maximum }\right\} \\
& \left\{V_{c c}=\beta \frac{C_{p}}{C_{n}-C_{p}} \frac{\alpha}{C_{p}} u_{M} \text { on strain minimum }\right\}
\end{aligned}
$$

The voltage and the displacement still vary proportionally, as given in Eq. (15), and the voltage inversion across the voltage source $V_{C C}$ leads to Eq. (37). The dissipated energy can be calculated readily from the energy cycle as shown in Fig. 4 and is given by Eq. (38) [20]:

$$
\left\{V_{m}-\beta \frac{C_{p}}{C_{n}-C_{p}} \frac{\alpha}{C_{p}} u_{M}=\gamma\left(V_{M}+\beta \frac{C_{p}}{C_{n}-C_{p}} \frac{\alpha}{C_{p}} u_{M}\right)\right\}
$$

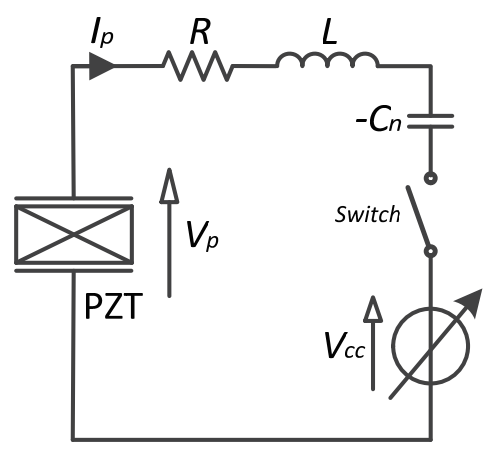

Fig. 10. Schematic diagram of SSDNCAV. PZT: piezoelectric patch.

$$
E_{t}=\frac{4 \alpha^{2}}{C_{p}}(1+\beta)\left(\frac{1+\gamma}{1-\gamma}\right) \frac{C_{n}}{C_{n}-C_{p}} u_{M}^{2}
$$

Eq. (38) shows an increase in the dissipated energy versus the previous SSD techniques as the energy cycle increases along the voltage axis of Fig. 4 .

The displacement is given by Eq. (39) under the assumption that it remains purely sinusoidal, where $\omega$ is the angular frequency of the driving force. The voltage on the piezoelectric patch can be expressed as the sum of two functions; one is the image of displacement or strain variation $V_{s t}$, while the other is a rectangular signal due to switching action $V_{s w}$, as shown in Fig. 11 and given by Eq. (40):

$$
\begin{aligned}
& u(t)=u_{M} \sin (\omega t+\varphi) \\
& V_{p}=V_{s t}+V_{s w}=\frac{\alpha}{c_{p}}(u+h)
\end{aligned}
$$

where $h$ is the crenel function defined in Eq. (41). In the case of periodic motion, $h$ is also periodic and can be expressed as a Fourier series, as shown in Eq. (42):

$$
\begin{gathered}
\left\{h=u_{M}(1+\beta)\left(\frac{1+\gamma}{1-\gamma}\right)\left(\frac{C_{n}}{C_{n}-C_{p}}\right) \operatorname{sign}(\dot{u})\right\} \\
\left\{\begin{array}{c}
h=u_{M}(1+\beta)\left(\frac{1+\gamma}{1-\gamma}\right)\left(\frac{C_{n}}{C_{n}-C_{p}}\right) \\
\sum_{n=0}^{\infty}\left(\frac{4}{\pi(2 n+1)} \cos ((2 n+1) \omega t+\varphi)\right)
\end{array}\right\} .
\end{gathered}
$$

Inserting Eq. (40) into Eq. (4) leads to Eq. (43). Only the first harmonic of the crenel can be considered, because the displacement is assumed to remain sinusoidal, which leads to the transfer function (44) linking the displacement to the excitation force:

$$
\left\{F=M \ddot{u}+\left(K_{E}+\frac{\alpha^{2}}{C_{p}}\right) u+C \dot{u}+\frac{\alpha^{2}}{C_{p}} h\right\}
$$

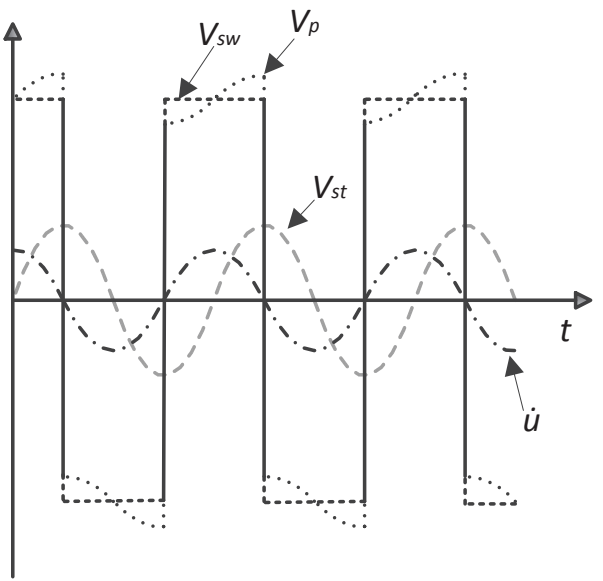

Fig. 11. Voltage on piezoelectric patch as the sum of two functions. 
Ehtesham Mustafa Qureshi Piezoelectric shunt damping by synchronized switching on negative capacitance and ...

$$
\left\{\widetilde{u} \frac{1}{\tilde{F}}=\frac{1}{\left(K_{E}+\frac{\alpha^{2}}{C_{p}}-M \omega^{2}+j\left(C \omega+\left(4 \alpha^{2} / \pi C_{p}\right)(1+\beta)(1+\gamma) /(1-\gamma)\left(C_{n} /\left(C_{n}-C_{p}\right)\right)\right)\right)}\right\}
$$

At the resonance frequency, still given by Eq. (8), the amplitude of displacement is given by Eq. (45) while the related SSDNCAV damping is given by Eq. (46):

$$
\begin{aligned}
& \left\{u_{M}=\frac{F_{M}}{\left(C \omega_{o}+\left(4 \alpha^{2} / \pi C_{p}\right)(1+\beta)(1+\gamma) /(1-\gamma)\left(C_{n} /\left(C_{n}-C_{p}\right)\right)\right)}\right\} \\
& \left\{A_{S S D N C A V}=20 \log \left(\frac{C \omega_{o}}{C \omega_{o}+\left(4 \alpha^{2} / \pi C_{p}\right)(1+\beta)(1+\gamma) /(1-\gamma)\left(C_{n} /\left(C_{n}-C_{p}\right)\right)}\right)\right\}
\end{aligned}
$$

Similar to the SSDVenh damping, Eq. (46) shows that the damping performance is insensitive to the amplitude of the applied force, thus keeping SSDNCAV stable. Also, the damping performance is dependent on the determination of proper value of negative capacitance $-C_{n}$. Keeping the derivations above in view, the value of negative capacitance $-C_{n}$ should be kept a little larger than the inherent capacitance $C_{p}$ of the piezoelectric patch; otherwise, the damping system will lose its stability. Although the electrical quality factor of the circuit limits the SSDNCAV damping performance, still the use of an adaptive voltage source in combination with the appropriate negative capacitance gives far better performance than the previous SSD techniques. Also, higher values of parameter $\beta$ render the previous theoretical developments invalid, because the displacement of higher-order modes cannot be neglected versus the fundamental one.

\section{Experimental Setup and Control Scheme}

The experimental set-up, consisting of all the necessary equipment, is shown in Fig. 12. It consists of a cantilever aluminum beam, clamped at one end on an electromagnetic exciter that generates a periodic force. One piezoelectric patch is bonded on the top surface and another one on the bottom surface of the cantilever beam near its clamped end. The upper piezoelectric patch is used for control purposes, while the one bonded on the bottom surface of the beam is used as a displacement sensor. The distance between the clamped end of the beam and the left edges of both the piezoelectric patches is $10 \mathrm{~mm}$. The poling direction of the piezoelectric patches is perpendicular to the beam while electromechanical coupling coefficient $k_{31}$ mainly drives the piezoelectric response. The electronic switch is driven by a DSP environment, based on a National Instruments PCIe7842R RIO device, using the Labview software. The electronic switch is turned by a trigger signal generated at the point of displacement extremum so that voltage on the piezoelectric control patch is inverted. The material properties and dimensions of the aluminum beam and piezoelectric patches are summarized in Table 1.

Five cases are considered for experiments: vibration without control, SSDI, SSDVenh, SSDNC, and SSDNCAV. A negative capacitance shunt can only be realized by

Table 1. Aluminum beam and piezoelectric patches properties and dimensions.

\begin{tabular}{lll}
\hline Aluminum & Size & $300 \times 40 \times 1 \mathrm{~mm}$ \\
beam & Elastic modulus & $E_{b}=70 \mathrm{GPa}$ \\
& Mass density & $\rho_{b}=2700 \mathrm{~kg} / \mathrm{m}^{3}$ \\
Piezoelectric & Size & $50 \times 40 \times 0.5 \mathrm{~mm}$ \\
patches & Elastic modulus & $E_{p}=66 \mathrm{GPa}$ \\
& Mass density & $\rho_{p}=7450 \mathrm{~kg} / \mathrm{m}^{3}$ \\
& Electromechanical coupling & $k_{31}=0.36$ \\
& coefficient & $C_{p}=67 \mathrm{nF}$ \\
\hline
\end{tabular}

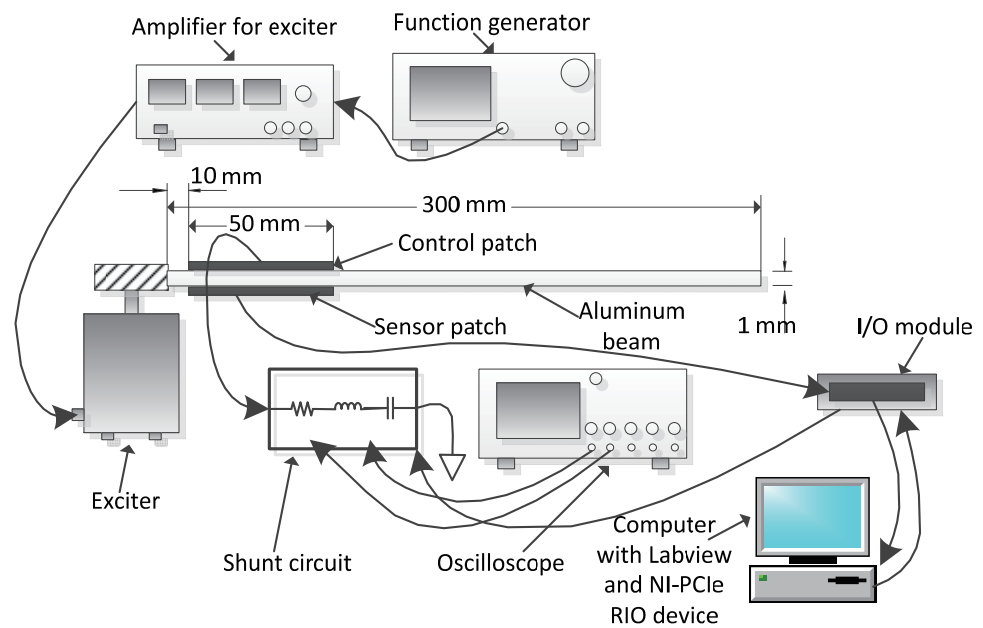

Fig. 12. Experimental setup. 
a negative impedance converter and is represented schematically in Fig. 13 [31]. It is an active storage element due to the use of an operational amplifier in combination with passive elements. The circuit's input impedance can be written as,

$$
Z_{\text {in }}=-\frac{1}{s R_{2} C_{g} / R_{1}}=-\frac{1}{s C_{n}}
$$

where $-C_{n}=-R_{2} C_{g} / R_{1}$ gives the negative capacitance. Thus, a

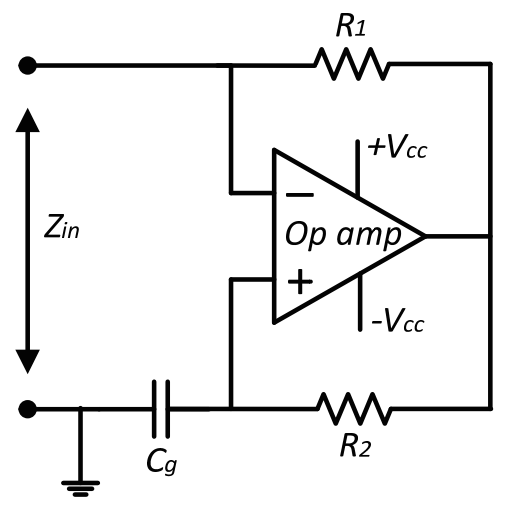

Fig. 13. Negative capacitance circuit schematic.

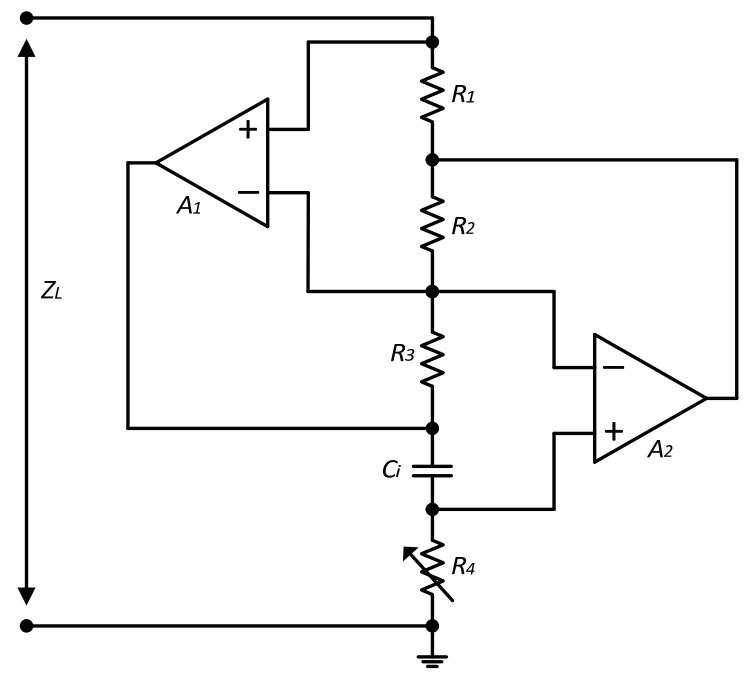

Fig. 14. Riordan's simulated grounded inductor. particular negative capacitance can be produced by varying the values of $R_{1}, R_{2}$ or $C_{g}$.

A synthetic inductance circuit is used instead of a coil inductor for convenience in tuning the value of inductance and the quality factor. This synthetic inductor is based on Riordan-type inductor [32] and is shown in Fig. 14. The input impedance $Z_{L}$ of the circuit is equivalent to:

$Z_{L}=S \frac{R_{1} R_{2} C_{i}}{R_{3}} R_{4}$

The equivalent inductance of the synthetic circuit $L_{I}=R_{1} R_{2} C_{i} R_{4} / R_{3}$, where $R_{4}$ is the adjustable resistance for inductance tuning. By carefully selecting the impedance values of the circuit elements of the synthetic inductor, the electrical quality factor $Q_{i}$ can be adjusted in a certain range. Table 2 lists the impedance values of all the circuit elements of the synthetic negative capacitance and inductance as used in the experiments.

\section{System Identification}

The identification of parameters of the piezoelectric structure considered is necessary to relate the identified model with the actual set-up. As the piezoelectric structure considered is more complex than the simple model described in Section 2, its various parameters cannot be simply obtained from the properties of piezoelectric patch. However, the structure presents all the features of a piezoelectric electromechanical device, as defined in Section 2. Thus, its properties can be identified from the experimental measurements, as summarized in Table 3 and Eq. (49) [20].

$$
\left\{\begin{array}{c}
\alpha=\lambda C_{p}, \quad K_{E}=\alpha \lambda \frac{f_{0}^{2}}{f_{1}^{2}-f_{0}^{2}}, \\
M=\frac{K_{E}}{4 \pi^{2} f_{0}^{2}}, \quad C=4 \pi \xi M f_{1}
\end{array}\right\}
$$

It is important to note that these identified parameters are valid for a narrow frequency band around a given resonance,

Table 2. Parameters of the synthetic negative capacitance and inductance circuits.

\begin{tabular}{lccc}
\hline & Symbol & $\begin{array}{c}\text { Negative } \\
\text { capacitance }\end{array}$ & Inductance \\
\hline Parameters of circuit & $R 1$ & $10 \mathrm{k} \Omega$ & $1 \mathrm{k} \Omega$ \\
elements & $R 2$ & $7 \mathrm{k} \Omega$ & $1 \mathrm{k} \Omega$ \\
& $R 3$ & & $1 \mathrm{k} \Omega$ \\
& $R 4$ & & $10 \mathrm{k} \Omega$ \\
& $C g$ & $100 \mathrm{nF}$ & \\
& $C_{i}$ & & $100 \mathrm{nF}$ \\
Equivalent impedance & $-C_{n}$ & $-70 \mathrm{nF}$ & \\
value & $L I$ & & $1 \mathrm{H}$ \\
\hline
\end{tabular}


Table 3. Measurements and model parameters.

\begin{tabular}{lcc}
\hline Short circuit resonance frequency & $f_{0}$ & $9.18 \mathrm{~Hz}$ \\
Open circuit resonance frequency & $f_{1}$ & $9.20 \mathrm{~Hz}$ \\
Open circuit damping coefficient & $\xi$ & 0.0088 \\
Piezo open circuit voltage to the beam free & $\lambda$ & $1146.67 \mathrm{~V} / \mathrm{m}$ \\
end displacement ratio & $\alpha$ & $7.68 \mathrm{E}-5 \mathrm{~N} / \mathrm{V}$ \\
Force factor & $C_{p}$ & $67 \mathrm{nF}$ \\
Clamped capacitance of the piezoelectric & $M$ & $0.006 \mathrm{~kg}^{-1}$ \\
element & $C$ & $0.0061 \mathrm{Nm}^{-1} \mathrm{~s}^{-1}$ \\
Equivalent mass & $K_{E}$ & $20.188 \mathrm{Nm}^{-1}$ \\
Equivalent inherent damping & $Q_{i}$ & 3.86 \\
Equivalent stiffness & $\gamma$ & 0.66 \\
Electrical quality factor & & \\
Voltage inversion coefficient &
\end{tabular}

which, in this case, is the first bending mode.

\section{Results and Discussion}

\subsection{Experiments under Single Frequency Excitation}

The cantilever beam is driven around its first resonance frequency of $9.2 \mathrm{~Hz}$. Without control and at the resonance frequency, the displacement of the beam is $7.5 \mathrm{~mm}$. Theoretical and experimental values of displacement amplitudes are kept same by optimizing the driving force in the simulation program. The exact values of the negative capacitance $-C_{n}$ and inductance $L_{I}$ are $-70 \mathrm{nF}$ and $1 \mathrm{H}$, respectively, (Table 2). Theoretical displacements are calculated on the basis of equations derived in Section 2. The voltage inversion coefficient $\gamma$ is 0.66 while the quality factor $Q_{i}$ of the circuit is 3.86 , which can be adjusted according to requirements by appropriately selecting the value of resistance and synthetic inductance in Eq. (13).

To evaluate the control performance of SSDNCAV

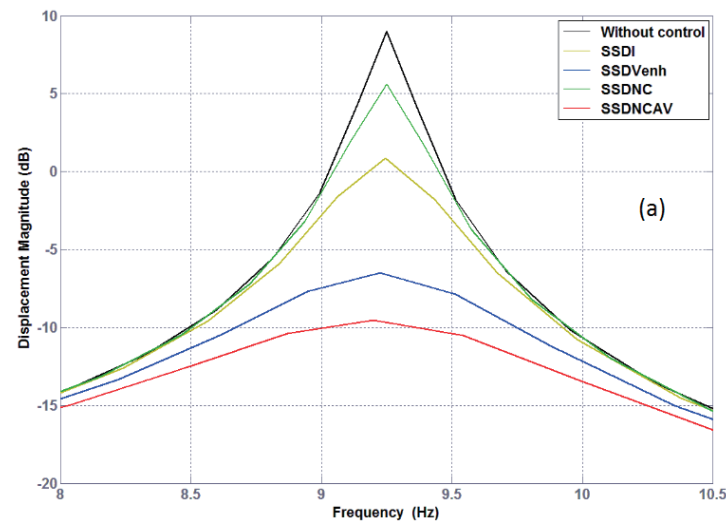

method in vibration control, the first-mode control of beam using different SSD techniques are carried out. The resulting theoretical and experimental frequency responses are plotted in Fig. 15, which are in close agreement with each other. The results were used to compare the control performance of SSDNCAV technique with the previous SSD techniques in the literature. The control performance expressed in displacement magnitude $(\mathrm{dB})$ is shown in Table 4. These results indicate that the experimental peak value of the spectrum is reduced by $18.45 \mathrm{~dB}$ with the SSDNCAV shunt network and by $15.43 \mathrm{~dB}$ with SSDVenh, while damping effects of $8.09 \mathrm{~dB}$ and $3.37 \mathrm{~dB}$ were achieved with SSDI and SSDNC for the same case. Thus, the control effect of SSDNCAV is better that the previous SSD techniques. This is attributable to the larger voltage magnification and more efficient voltage inversion in SSDNCAV versus the previous SSD techniques.

Figure 16 shows the experimental curves (a)-(e) of transient displacement responses of the cantilever beam for different SSD techniques. Table 5 lists the corresponding settling times reached within $2 \%$ of the steady state position

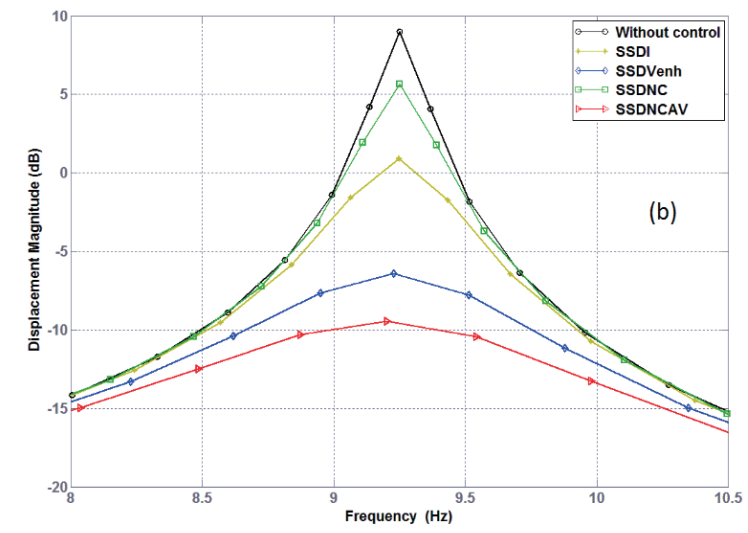

Fig. 15. Theoretical (a) and experimental (b) frequency responses of different SSD techniques. 
Int'I J. of Aeronautical \& Space Sci. 15(4), 396-411 (2014)

Table 4. Control performance in terms of displacement magnitude (dB) of different SSD techniques.

\begin{tabular}{lcc}
\hline & $\begin{array}{c}\text { Peak value (upper) } \\
\text { and reduction (lower) }(\mathrm{dB}) \\
\text { (Theoretical results) }\end{array}$ & $\begin{array}{c}\text { Peak value (upper) } \\
\text { and reduction (lower) }(\mathrm{dB}) \\
\text { (Experimental results) }\end{array}$ \\
\hline Without control & 9.01 & 9.01 \\
\multirow{2}{*}{ SSDI } & - & - \\
& 0.87 & 0.92 \\
SSDVenh & 8.14 & 8.09 \\
& -6.50 & -6.42 \\
SSDNC & 15.51 & 15.43 \\
& 5.61 & 5.64 \\
SSDNCAV & 3.4 & 3.37 \\
& -9.53 & -9.44 \\
& 18.54 & 18.45 \\
\hline
\end{tabular}

for comparison. Curve (a) is the free response of the beam when the control system is turned off. When the control system is turned on, the percent reduction in settling time of SSDNCAV is $93.1 \%$, versus $91.3 \%, 59.9 \%$, and $30.2 \%$ for
SSDVenh, SSDI, and SSDNC respectively. Thus, SSDNCAV demonstrated better transient displacement responses than the previous SSD techniques.

Appropriate determination of parameters $\beta$ and $-C_{n}$
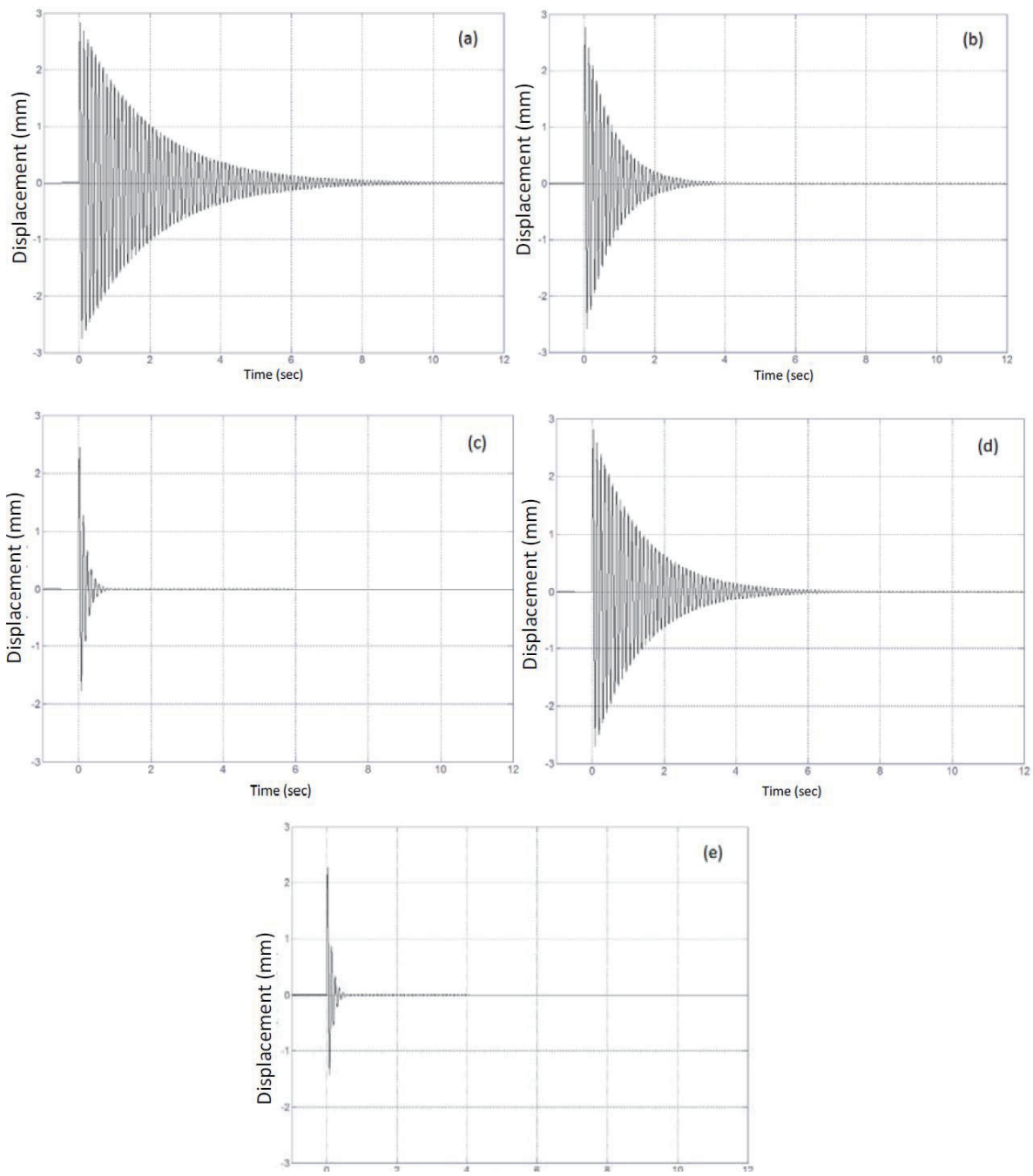

Fig. 16. Transient displacement responses of the beam for different SSD techniques: (a) without control; (b) SSDI; (c) SSDVenh; (d) SSDNC; (e) SSDNCAV. 
Table 5. Settling time of experimental transient displacement response of the beam for different SSD techniques.

\begin{tabular}{lc}
\hline & Settling time $(\mathrm{sec})$ \\
\hline Without & 11.3 \\
control & \\
SSDI & 4.53 \\
SSDVenh & 0.98 \\
SSDNC & 7.88 \\
SSDNCAV & 0.77 \\
\hline
\end{tabular}

are important to ensure the stability and effectiveness of SSDVenh and SSDNC, respectively [20, 27]. Fig. 17 shows attenuation of SSDNCAV shunt damping at the resonance frequency versus $\beta$ and $-C_{n}$. The curve (a) of Fig. 17 is obtained by keeping the negative capacitance constant at $-C_{n}=-70 \mathrm{nF}$, while in curve (b), a constant value of the proportional coefficient $\beta=2.7$ is used. From Fig. 17(a), it is clear that for $\beta=2.7$, the SSDNCAV technique is stable regardless of the amplitude of the driving force. Selecting a higher value of $\beta$ improves the damping performance of SSDNCAV (i.e., the damping reaches $-25 \mathrm{~dB}$ for $\beta=8$, while keeping the system stable). The parameter $\beta$ can be considered as a feedback gain, allowing for continuous adjustment of the control force amplitude to the vibration level. Theoretically, this closed loop process is stable for any value of $\beta$. However, as is usually the case, for large values of feedback gains, which limit the modulus margin and render the system instable when disturbances due to noise or harmonics are important as compared with the excited mode. Experimentally, the noise and electronic implementation imperfections reach the modulus margin for $\beta$ greater than 8 , as shown in Fig. 17(a). Remarkably, under the stability limit, the system is stable and the damping is constant for any amplitude of the exciting force.
Analysis of Fig. 17(b) shows that damping performance of the SSDNCAV technique depends on the absolute value of negative capacitance $-C_{n}$. The closer it gets to the inherent capacitance $C_{p}$ of the piezoelectric patch, the better vibration damping it exhibits. Its effectiveness fades along with the increase in the value. Choosing a value of $-C_{n}$ lower than $C_{p}$ can destabilize the structure. Although a lower value of $-C_{n}$ can give larger vibration attenuation but the destabilization factor must be taken into account before choosing the value for negative capacitance. In fact, in the SSDNCAV technique, it is observed that keeping the value of negative capacitance slightly greater than the inherent capacitance of the piezoelectric patch guaranteed the effectiveness of the damping performance of SSDNCAV, without hurting its stability.

In the experiments, the electrical quality factor $Q_{i}$ could not be increased beyond 3.86, which limits the damping performance of the SSDI technique. The impact of $Q_{i}$ is not so prominent in SSDVenh and SSDNCAV due to the adaptive nature of these techniques, which compensated for the lower value of $Q_{i}$. In fact, the best damping performance was achieved by the SSDNCAV technique due to voltage inversion and magnification via the capacitance transient charging, electrical resonance, and adaptation of piezoelectric patch voltage according to the vibration amplitude of the structure.

\subsection{Experiments under Broadband Excitation}

The same experimental set-up shown in Fig. 12 was used for broadband vibration attenuation. The cantilever beam is driven with a short sine sweep signal to excite frequencies on a $500 \mathrm{~Hz}$ frequency band. This frequency band was chosen because it contained the first four resonance frequencies of the cantilever beam. These resonance frequencies are $9.2 \mathrm{~Hz}$,

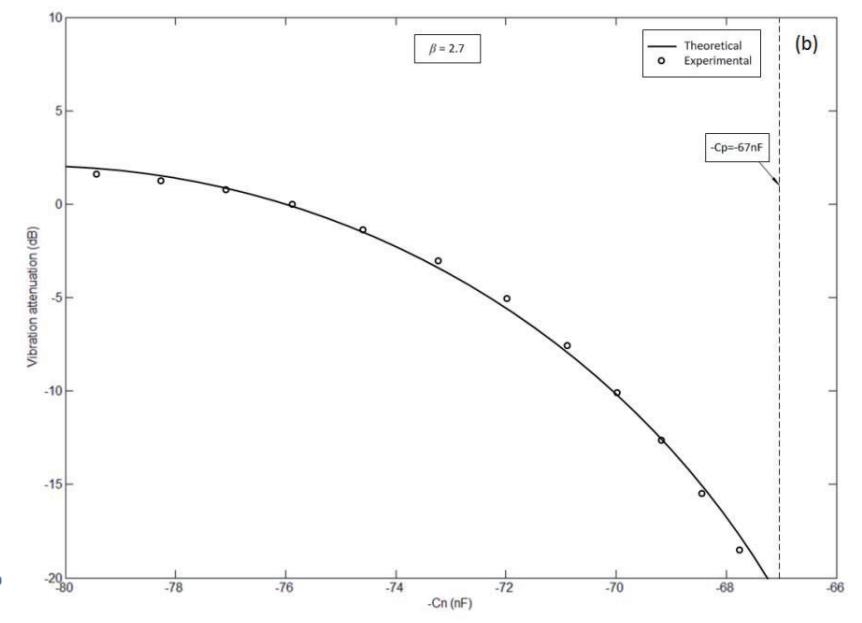

Fig. 17. Theoretical and experimental SSDNCAV damping versus: (a) $\beta$ at constant $-C_{n}$; and (b) $-C_{n}$ at constant $\beta$. 
57.2 Hz, 160.4 Hz, and 314.3 Hz, respectively. Experiments under broadband excitation were performed to show the effectiveness of the SSDNCAV technique in controlling the vibration of structures subjected to more realistic environmental disturbances as is usually the case in the aerospace field.

During the experiments, the sensor patch generates the sensing voltage $V_{s}$, which is used by the digital signal processor (DSP) to drive the electronic switch according to the displacement extrema. Basically, the electronic switch connects or disconnects the piezoelectric patch from the shunt circuit for control purpose. The adaptive control in both SSDVenh and SSDNCAV involves setting the amplitude of the continuous voltage source $V_{C C}$ to be proportional to the amplitude of $V_{s}$ [20] (see Eq. (50)). $V_{C C}$ is actually the output of a basic operational amplifier driven by the DSP. In the experiments, the value of the optimal gain coefficient $\beta$ was 2.7 and it needed to be fitted only once:

$$
V_{c c}=\beta V_{s}
$$

Five cases are considered in the experiment: vibration without control, vibration control with SSDI, SSDVenh, SSDNC, and SSDNCAV. To evaluate the control performance, the power spectral density (PSD) estimates (in $\mathrm{dB} / \mathrm{Hz}$ ) of the
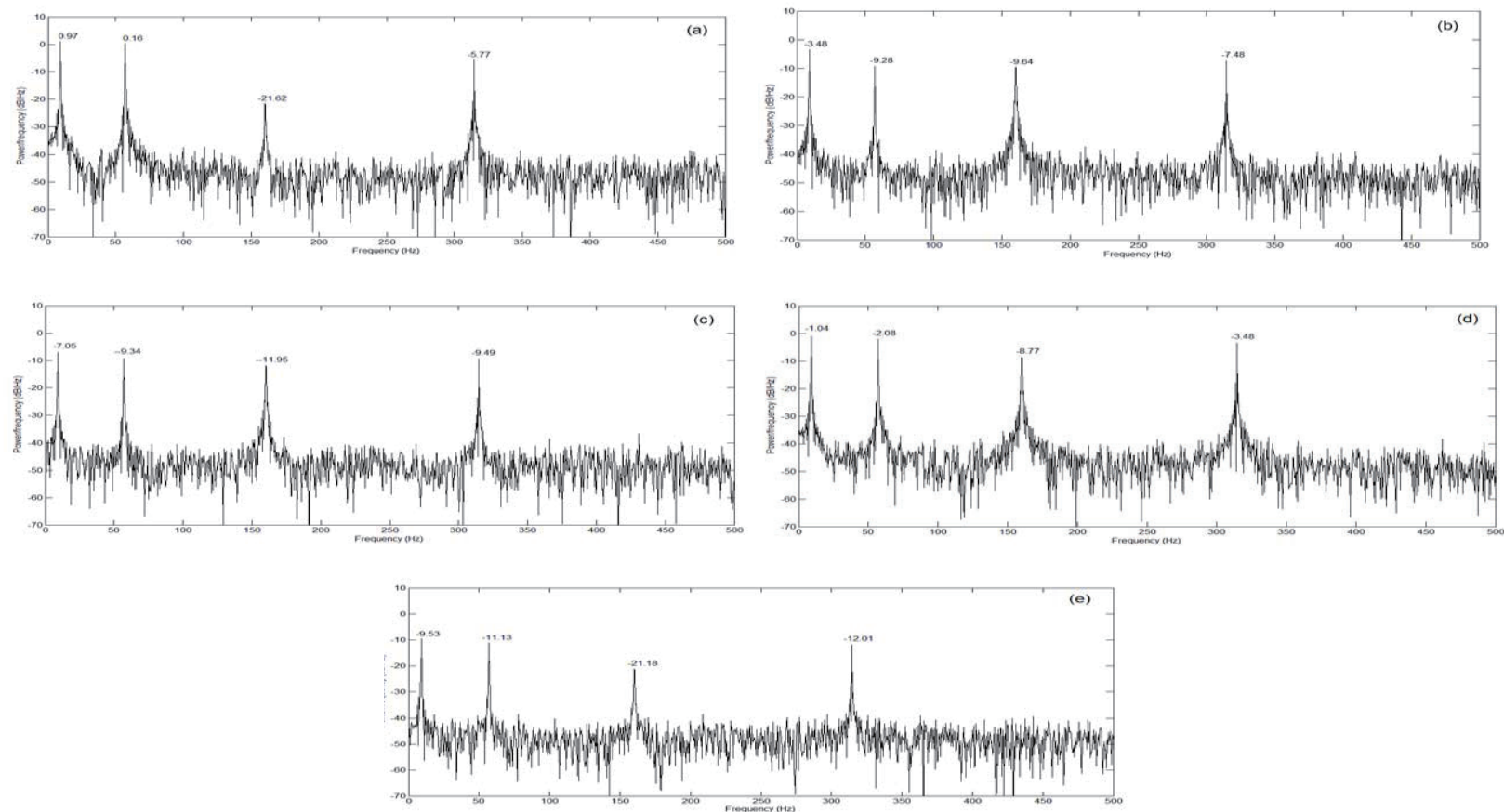

Fig. 18. Power spectral density estimates of the response of beam for different SSD techniques: (a) without control; (b) SSDI; (c) SSDVenh; (d) SSDNC; (e) SSDNCAV.

Table 6. Power spectral density estimates $(\mathrm{dB} / \mathrm{Hz})$ of the response of the beam for different SSD techniques.

\begin{tabular}{|c|c|c|c|c|c|}
\hline & $\begin{array}{l}\text { First mode, } \\
\text { Peak value } \\
\text { (upper) } \\
\text { and reduction } \\
\text { (lower) }\end{array}$ & $\begin{array}{l}\text { Second mode, } \\
\text { Peak value } \\
\text { (upper) } \\
\text { and reduction } \\
\text { (lower) }\end{array}$ & $\begin{array}{l}\text { Third mode, } \\
\text { Peak value } \\
\text { (upper) } \\
\text { and reduction } \\
\text { (lower) }\end{array}$ & $\begin{array}{l}\text { Fourth mode, } \\
\text { Peak value } \\
\text { (upper) } \\
\text { and reduction } \\
\text { (lower) }\end{array}$ & $\begin{array}{c}\text { Average } \\
\text { power }\end{array}$ \\
\hline Without control & $\begin{array}{c}0.97 \\
-\end{array}$ & $\begin{array}{c}0.16 \\
-\end{array}$ & $\begin{array}{c}-21.62 \\
-\end{array}$ & $\begin{array}{c}-5.77 \\
-\end{array}$ & 1.37 \\
\hline SSDI & $\begin{array}{l}-3.48 \\
4.45\end{array}$ & $\begin{array}{l}-9.28 \\
9.44\end{array}$ & $\begin{array}{l}-9.64 \\
-11.98\end{array}$ & $\begin{array}{c}-7.48 \\
1.71\end{array}$ & 0.53 \\
\hline SSDVenh & $\begin{array}{r}-7.05 \\
8.02\end{array}$ & $\begin{array}{l}-9.34 \\
9.50\end{array}$ & $\begin{array}{l}-11.95 \\
-9.67\end{array}$ & $\begin{array}{r}-9.49 \\
3.72\end{array}$ & 0.31 \\
\hline SSDNC & $\begin{array}{c}-1.04 \\
2.01\end{array}$ & $\begin{array}{l}-2.08 \\
2.24\end{array}$ & $\begin{array}{l}-8.77 \\
-12.85\end{array}$ & $\begin{array}{l}-3.48 \\
-2.29\end{array}$ & 1.17 \\
\hline SSDNCAV & $\begin{array}{c}-9.53 \\
10.5\end{array}$ & $\begin{array}{r}-11.13 \\
11.29\end{array}$ & $\begin{array}{l}-21.18 \\
-0.44\end{array}$ & $\begin{array}{c}-12.01 \\
6.24\end{array}$ & 0.16 \\
\hline
\end{tabular}


response signal of the beam is obtained for the five cases, as shown in Fig. 18 (a)-(e). The first four resonance modes are clearly visible in these plots. Table 6 lists the corresponding PSD values of the four modes, along with the average power of the response signal for different SSD techniques. Without control, the first mode $(9.2 \mathrm{~Hz})$ exhibits the peak value, principally because it is the highest mode. Considering the first resonance mode, vibration attenuation of $10.5 \mathrm{~dB} / \mathrm{Hz}$ is shown by the SSDNCAV technique, followed by SSDVenh (8.02 dB/Hz), SSDI (4.45 dB/Hz), and SSDNC $(2.01 \mathrm{~dB} / \mathrm{Hz})$. The same trend was observed for the second and fourth modes. However, for the third mode, at $160.4 \mathrm{~Hz}$, the peak values increase for all the SSD techniques, with the greatest value observed for the SSDNC technique. In this case, also, SSDNC emerged as the least effective control technique among the different SSD technique used. The increase in the magnitude of $160.4 \mathrm{~Hz}$ line is attributable to it corresponding to the resonance frequency of the structure as well as to an odd multiple of the first resonance frequency.

Table 6 also lists the average power $(\mathrm{dB} / \mathrm{Hz})$ of the response signal of the beam for different SSD techniques. The average power can also be considered as an indicator of the control performance of different SSD techniques, with the least value corresponding to the best control performance. From Table 6 it is clear that SSDNCAV exhibited the lowest average power, followed by SSDVenh, SSDI, and SSDNC, thus creating greater electro-mechanical energy conversion and significant vibration attenuation over a broad spectrum range.

\section{Conclusions}

A new SSD technique, called SSDNCAV, has been proposed, combining the effects of negative capacitance and electrical resonance in the switching shunt circuit for structural vibration control using a shunted piezoelectric patch. Its effectiveness in vibration control of a cantilever beam was investigated experimentally and compared with the previous SSD techniques. The expression of the vibration attenuation at the resonance frequency for SSDNCAV was derived based on a unifying mechanical model. The problem of stability observed in previous SSD techniques was also addressed by the adaptive control of the continuous voltage source output according to the vibration level. The continuous voltage source, which is the output of a basic operational amplifier, was simply set proportional to the output voltage of the sensor patch. It was shown that SSDNCAV gave a stable damping regardless of the amplitude of the excitation force (up to $25 \mathrm{~dB}$ when $\beta=8$ ). The influence of negative capacitance on damping performance of SSDNCAV technique was also investigated. It was found that the damping performance increased when the value of negative capacitance approached the value of the piezoelectric patch capacitance. In fact, a value slightly greater that the piezoelectric patch capacitance, guaranteed the effectiveness of the damping performance of SSDNCAV, without hurting its stability. Experimental results also demonstrated the broadband damping capabilities of SSDNCAV technique, as compared with previous SSD techniques, demonstrating its effectiveness in controlling vibration under actual environmental conditions.

\section{Acknowledgments}

This work was sponsored by Qing Lan Project and a Project funded by the PAPD. The authors are also grateful to the Fundamental Research Funds for the Central Universities (No. NS2013010).

\section{References}

[1] Moheimani, S.O.R., "A survey of recent innovations in vibration damping and control using shunted piezoelectric transducers", IEEE Transactions on Control Systems Technology, Vol. 11, 2003, pp. 482-494. DOI:10.1109/ TCST.2003.813371

[2] Davis, C.L. and Lesieutre, G.A., "A modal strain energy approach to the prediction of resistivity shunted piezoceramic damping", Journal of Sound and Vibration, Vol. 184, No. 1 ,1995, pp. 129-139. DOI: 10.1006/jsvi.1995.0308

[3] Forward, R.L., "Electronic damping of vibrations in optical structures”, Applied Optics, Vol.18, No. 5, 1979, pp. 690-697. DOI: 10.1364/AO.18.000690

[4] Hagood, N.W. and von Flotow, A., "Damping of structural vibrations with piezoelectric materials and passive electrical networks", Journal of Sound and Vibration, Vol. 146, No. 2, 1991, pp. 243-268. DOI: 10.1016/0022-460X(91)90762-9

[5] Qiu, J., Ji, H. and Zhu, K., "Semi-active vibration control using piezoelectric actuators in smart structures", Frontiers of Mechanical Engineering in China, Vol. 4, No. 9, 2009, pp. 242-251. DOI: 10.1007/s11465-009-0068-z

[6] Wang, Y. and Inman, D.J., "A survey of control strategies for simultaneous vibration suppression and energy harvesting via piezoceramics", Journal of Intelligent Material Systems and Structures, Vol. 23, No. 18, 2012, pp. 2021-2037. DOI: $10.1177 / 1045389 X 12444485$

[7] Hagood, N.W. and Crawley, E.F., "Experimental 
investigations of passive enhancement of damping space structures", Journal of Guidance, Control and Dynamics, Vol. 14, No. 6, 1991, pp. 1100-1109.

DOI: $10.2514 / 3.20763$

[8] Hollkamp, J.J., "Multimodal passive vibration suppressionwith piezoelectricmaterialsandresonantshunts", Journal of Intelligent Material Systems and Structures, Vol. 5, No. 1, 1994, pp. 49-56. DOI: 10.1177/1045389X9400500106

[9] Law, H.H., Rossiter, P.L., Simon, G.P. and Koss, L.L., "Characterization of mechanical vibration damping by piezoelectric materials", Journal of Sound and Vibration, Vol. 197, No. 4, 1996, pp. 489-513. DOI:10.1006/jsvi.1996.0544

[10] Behrens, S., Moheimani, S.O.R. and Fleming, A.J.,"Multiple mode current flowing passive piezoelectric shunt controller", Journal of Sound and Vibration, Vol. 266, Issue 5, 2003, pp. 929-942. DOI:10.1016/S0022460X(02)01380-9

[11] Richard, C., Guyomar, D., Audigier, D. and Ching, G., "Semi-passive damping using continuous switching of a piezoelectric device", In Proc. SPIE Conf. Passive Damping Isolation, Newport Beach, CA, Vol. 3672, 1999, pp. 104-111. DOI:10.1117/12.349773

[12] Clark, W.W., "Vibration control with state-switched piezoelectric materials", Journal of Intelligent Material Systems and Structures, Vol. 11, Issue 4, 2000, pp. 263-271. DOI: 10.1106/18CE-77K4-DYMG-RKBB

[13] Richard, C., Guyomar, D. and Audigier, D., et al., "Enhanced semi-passive damping using continuous switching of a piezoelectric device on an inductor", In Proc. of SPIE Smart Structures and Materials 2000: Damping and Isolation, Vol. 3989, 2000, pp. 288-299. DOI:10.1117/12.384569

[14] Guyomar, D., Richard, C. and L. Petit, L., "Non-linear system for vibration damping", 142nd Meeting of Acoustical Society of America, Fort Lauderdale, FL, 2001.

[15] Cunefare, K.A., "State-switched absorber for vibration control of point-excited beams", Journal of Intelligent Material Systems and Structures, Vol. 13, Issue 2-3, 2002, pp. 97-105. DOI:10.1177/104538902761402495

[16] Onoda, J., Makihara, K. and Minesugi, K., "Energyrecycling semi-active method for vibration suppression with piezoelectric transducers", AIAA Journal, Vol. 41 No. 4, 2003, 711-719. DOI: $10.2514 / 2.2002$

[17] Corr, L.R. and Clark, W.W., "A novel semi-active multimodal vibration control law for a piezoceramic actuator", Journal of Vibration and Acoustics, Vol. 125, Issue 2, 2003, pp. 214-222. DOI:10.1115/1.1547682

[18] Petit, L., Lefeuvre, E., Richard, C. and Guyomar, D., "A broadband semipassive piezoelectrictechniquefor structural damping", Proceedings of SPIE International Symposium on
Smart Structure Materials: Damping and Isolation, Vol. 5386, 2004, pp. 414-425. DOI:10.1117/12.532716

[19] Lefeuvre, E., Badel, A. and Petit, L., et al., "Semipassive piezoelectric structural damping by synchronized switching on voltage sources", Journal of Intelligent Material Systems and Structures, Vol. 17, Issue 8-9, 2006, pp. 653-660. DOI: 10.1177/1045389X06055810

[20] Badel, A., Sebald, G. and Guyomar, D., et al., "Piezoelectric vibration control by synchronized switching on adaptive voltage sources: towards wideband semi-active damping", Journal of the Acoustical Society of America, Vol. 119, No. 5, 2006, pp. 2815-2825. DOI: 10.1121/1.2184149

[21] Neubauer, M. and Wallaschek, J., "Analytical and experimental investigation of the frequency ratio and switching law for piezoelectric switching techniques", Smart Materials and Structures, Vol.17, Issue 3, 2008, pp. 035003 (9pp). DOI:10.1088/09641726/17/3/035003

[22] Ji, H., Qiu, J. and Badel, A., et al., "Semi-active vibration control of a composite beam by adaptive synchronized switching on voltage sources based on LMS algorithm", Journal of Intelligent Material Systems and Structures, Vol. 20, Issue 8, 2009, pp. 939-947. DOI: 10.1177/1045389X08099967

[23] Ji, H., Qiu, J. and Zhu, K., et al., "Multi-modal vibration control using a synchronized switch based on a displacement switching threshold", Smart Materials and Structures, Vol. 18, Issue 3, 2009, pp. 035016 (8 pp). DOI:10.1088/0964$1726 / 18 / 3 / 035016$

[24] Ji, H., Qiu, J., Zhu, K. and Badel, A., “Two-mode vibration control of a beam using nonlinear synchronized switching damping based on the maximization of converted energy", Journal of Sound and Vibration, Vol. 329, Issue 14, 2010, pp. 2751-2767. DOI:10.1016/j.jsv.2010.01.012

[25] Ji, H., Qiu, J. and Xia, P., "Analysis of energy conversion in two-mode vibration control using synchronized switch damping approach", Journal of Sound and Vibration, Vol. 330, Issue 15, 2011, pp. 3539-3560. DOI: 10.1016/j.jsv.2011.03.004

[26] Qureshi, E.M., Shen , X. and Chen J.J., "Vibration control laws via shunted piezoelectric transducers: A review", International Journal of Aeronautical and Space Sciences, Vol. 15, No. 1, 2014, pp. 1-19. DOI:10.5139/IJASS.2014.15.1.1

[27] Ji, H., Qiu, J., Cheng, J. and Inman, D., "Application of a negative capacitance circuit in synchronized switch damping techniques for vibration suppression", Journal of Vibration and Acoustics, Vol. 133, Issue 4, 2011, pp. 0410151-10. DOI:10.1115/1.4003146

[28] Ji, H., Qiu, J., Badel, A. and Zhu, K., "Semi-active vibration control of a composite beam using an adaptive SSDV approach", Journal of Intelligent Material Systems and Structures, Vol. 20, Issue 4, 2009, pp. 401-412. DOI: 10.1177/1045389X08095182 
Ehtesham Mustafa Qureshi Piezoelectric shunt damping by synchronized switching on negative capacitance and ...

[29] Mokrani, B., Rodrigues, G., Ioan, B., Bastaits, R. and Preumont, A., "Synchronized switch damping on inductor and negative capacitance", Journal of Intelligent Material Systems and Structures, Vol. 23, No. 18, 2012, pp. 2065-2075. DOI: $10.1177 / 1045389 X 11433493$

[30] Han, X., Neubauer, M. and Wallaschek, J., “Improved piezoelectric switch shunt damping technique using negative capacitance", Journal of Sound and Vibration, Vol. 332, Issue 1, 2013, pp. 7-16. DOI: 10.1016/j.jsv.2012.08.001

[31] Horowitz, P. and Hill, W., The Art of Electronics, Cambridge University Press, New York, 1996.

[32] Riodan, R.H.S., "Simulated inductors using differential amplifiers", Electronic Letter, Vol. 3, No. 2, 1967, pp. 50-51. DOI: 10.1049/el: 19670039 